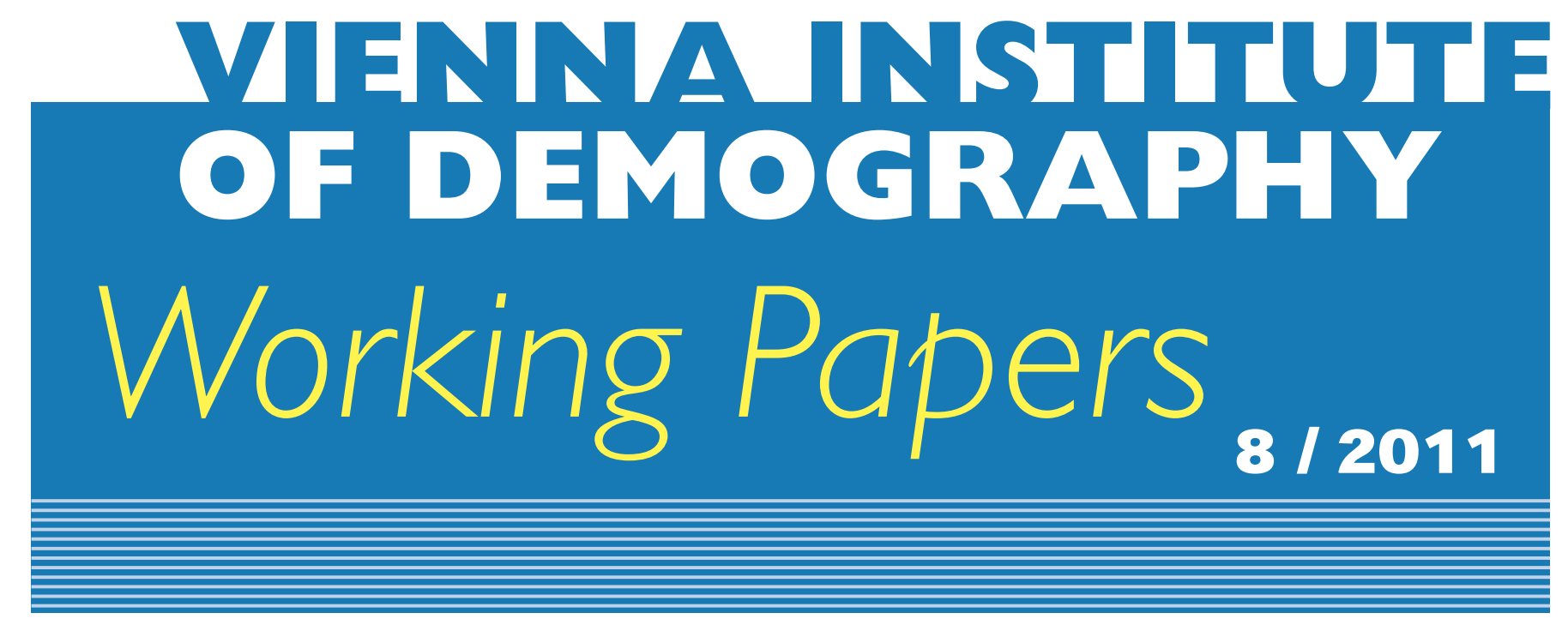

Dalkhat M. Ediev

\title{
At Modal Age at Death, the Hazard Rate is Determined by its Derivative
}

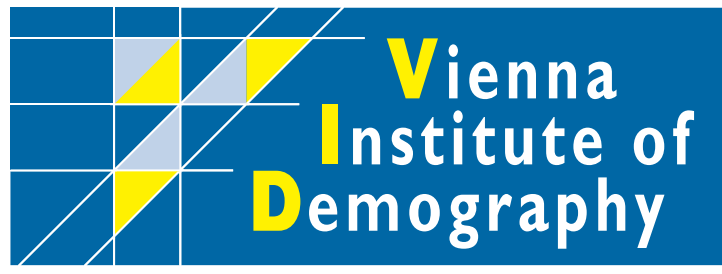

Vienna Institute of Demography Austrian Academy of Sciences

Wohllebengasse I2-I4

A-I040Vienna $\cdot$ Austria

E-Mail:vid@oeaw.ac.at

Website: www.oeaw.ac.at/vid

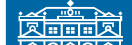

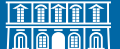




\begin{abstract}
The relation between the hazard rate and its derivative at modal age at death, an equivalent to which has been featured by Pollard (1991), Canudas-Romo (2008), Thatcher et al. (2010), and Tuljapurkar and Edwards (2011), is presented as a handy tool in studying mortality compression in its period and cohort dimensions. Our analytical findings indicate birth cohorts to differ substantially from the period life tables with respect to the distribution of deaths around the mode. Empirical results support theoretical predictions and show that previously reported effect of compression of deaths above the mode might be a feature of period life tables and not of the cohort mortality schedules. Our results are also useful in computing the modal age at death and sensitivity analysis.
\end{abstract}

\title{
Keywords
}

Modal age, hazard rate, mortality compression, rectangularization, tempo effect, cohort mortality.

\section{Author}

Dalkhat M. Ediev is research scientist at the Vienna Institute of Demography (Austrian Academy of Sciences), Wittgenstein Centre for Demography and Global Human Capital.

E-mail: dalkhat.ediev@,oeaw.ac.at

\section{Acknowledgements}

The author is thankful to anonymous reviewers for their comments on an earlier draft submitted to Demographic Research. 


\title{
At Modal Age at Death, the Hazard Rate is Determined by its Derivative
}

\author{
Dalkhat M. Ediev
}

\section{The Key Relationship}

Let $l(x)$ be the proportion surviving from birth to age $x$ (the survival function) and $d(x)=-\frac{d}{d x} l(x)$ the density function of the distribution of individuals by age at death. Then, at the modal age at death $x=M>0$, when $d(x)$ has a (local) maximum, the value and derivative of the force of mortality $\mu(x)=\frac{d(x)}{l(x)}$ are linked by a family of relations:

$$
\sqrt[2-\alpha]{\frac{1}{1-\alpha} \frac{d}{d x} \mu^{1-\alpha}(x)}=\mu(x),(1)
$$

where $\alpha \neq 1,2$ is parameter determining a member from the family. (The relations do not apply to the trivial mode at $x=0$.)

\section{The Proof}

$$
\frac{d}{d x} \mu(x)=\frac{d}{d x}\left(\frac{d(x)}{l(x)}\right)=\frac{\frac{d}{d x} d(x)}{l(x)}-\frac{d(x) \frac{d}{d x} l(x)}{l^{2}(x)}=\frac{\frac{d}{d x} d(x)}{l(x)}+\frac{d(x) d(x)}{l^{2}(x)}=\frac{\frac{d}{d x} d(x)}{l(x)}+\mu^{2}(x) .
$$

At modal age $x=M>0$, where the life-table functions are differentiable, $\frac{d}{d x} d(x)=0$. Hence, the key relationship at $\alpha=0$. The general relation (1) is obtained by multiplying (2) by $\mu^{-\alpha}(x)$ and noting that $\mu^{-\alpha}(x) \frac{d}{d x} \mu(x)=\frac{1}{1-\alpha} \frac{d}{d x} \mu^{1-\alpha}(x)$.

\section{Some Other Relationships That Are Closely Linked to, and Can Be Readily Derived from, the Key Relationship}

Two particular members of the family (1) were featured in the literature. Namely, at $\alpha=0$,

$$
\frac{d}{d x} \mu(x)=\mu^{2}(x) ;(3)
$$

and, at $\alpha \rightarrow 1$,

$$
\frac{d}{d x} \ln \mu(x)=\mu(x),(4)
$$

which limit is easy to obtain dividing (3) by $\mu(x)$. (See the next section for the history of these relations.)

Although all members of the family (1) are formally equivalent, one particular choice for the parameter $\alpha$, at which the left-hand side in (1) is approximately constant around the mode, provides some practical convenience in sensitivity analysis (concrete examples are given in the applications section below). To find general solution for $\alpha$ fulfilling the choice, one can equate to zero the derivative of the expression in the left-hand side of Eq. (1):

$$
0=\frac{d}{d x}\left[\frac{1}{1-\alpha} \frac{d}{d x} \mu^{1-\alpha}(x)\right]=\frac{d}{d x}\left[\mu^{-\alpha}(x) \frac{d}{d x} \mu(x)\right]=(-\alpha) \mu^{-\alpha-1}(x)\left(\frac{d}{d x} \mu(x)\right)^{2}+\mu^{-\alpha}(x) \frac{d^{2}}{d x^{2}} \mu(x) \text { (5) }
$$

at $x=M$. Substituting (3), this leads to the solution: 


$$
\alpha=\alpha^{*}=\frac{\frac{d^{2} \mu}{d x^{2}}(M)}{\mu^{3}(M)} .
$$

One can also express this solution in terms of distributional characteristics of deaths rather than in terms of the hazard rate. We present the expression without derivation here:

$$
\alpha^{*}=2+l^{2}(M) \frac{\frac{d^{2}}{d x^{2}} d(M)}{d^{3}(M)} .
$$

(One may come to the expression by deriving the second derivative from (2) and taking Eqs. (3), (6) into account.)

Aside from the applications to sensitivity analysis presented below, parameter $\alpha^{*}$ is useful in describing the mortality pattern around the most typical age at adult death and, hence, in specifying the type of the adult mortality model consistent with data. The Gompetz 'law of mortality' $\mu(x)=a e^{b x}$ yields, e.g., $\alpha^{*}=1$ at any combination of its parameters. The normal distribution of the age at death (assumed in studies featuring the modal age at death), $d(x) \sim \frac{1}{2 \pi \sigma^{2}} e^{-\frac{(x-M)^{2}}{2 \sigma^{2}}}$ suggests another value $\alpha^{*}=2-\frac{\pi}{2} \approx 0.43$ at any combination of its parameters. Meanwhile, empirical mortality schedules (we used data for currently lowmortality countries from the Human Mortality Database (2011)) disagree with both these models and yield $\alpha^{*}$ mainly distributed between 0.4 and 1 . In period life tables, $\alpha^{*} \approx 0.75$ on average since 1950 s without notable time trend; in cohort data, $\alpha^{*} \approx 0.83$ on average after 1950 s with tendency to increase after 1970s. As a rough estimate, we take $\alpha^{*} \approx 0.8$ for further calculations.

\section{History of the Discovery of the Relationship}

Relation in the form of Eq. (4) was first derived, in the context of the Gompertz law of mortality, by Pollard (1991). It was rediscovered later as a general relation by Canudas-Romo (2008; Schoen 2006 cites a similarly titled unpublished manuscript by Canudas-Romo from 2005) and Thatcher et al. (2010; the authors cite their own conference submission from 2006). The derivative of the logarithm of the hazard rate in (4) is often referred to as the life table ageing rate (Carey and Liedo 1995). The ageing rate is particularly important a parameter in the Gompertz model of mortality, where it is age-independent. As Eq. (4) states, at the mode, the aging rate equals the hazard rate. More recently, Tuljapurkar and Edwards (2011) presented the relationship in form (3) in this journal.

The motivation to study the modal age at death comes from two long-lasting lines of research which seem to have merged recently: one concerning the 'normal' distribution of deaths and the other concerning the mortality compression. The former tradition originates from Quetelet and Lexis and was more recently revived by Kannisto (for a review, see Cheung and Robine 2007). Lexis (1878) suggested that the modal age at (adult) death, $M$, represents a typical 'natural' duration of human life and that durations of life exceeding $M$ follow the normal distribution (as opposed to the durations of life below $M$, where child and premature deaths top up the normally distributed 'natural' deaths). Based on modern data, Kannisto $(2000,2001)$ supported Lexis's assumption of normality of the distribution of deaths above the mode and advocated usage of the mode as an indicator of human longevity, supplementary to 'life expectancy at birth'. He also proposed to use the standard deviation of life durations above the mode as an indicator of mortality compression (Kannisto 2001) thereby linking the concepts of the mode and 'natural' deaths to another long-lasting concept 
of mortality compression (Fries 1980; see Thatcher et al. 2010 for a recent review). It is worthwhile noting, in this context, that Wilmoth and Horiuchi (1999) also proposed an indicator of mortality compression, the Fastest Decline (FD), linked to the modal age (FD is the height of the deaths' density function at the modal age, $d(M)$ ). While indicating its better performance in terms of correlation to other alternative measures of mortality compression, they nonetheless opted for the Interquartile Range as a more convenient indicator.

\section{Possible Applications}

As a general analytical relation, Eq. (1) may have variety of applications when it comes to study the modal age at death. Here, we highlight three particular applications: to determining the mode and compression of deaths; analysing effects of small mortality change; and studying tempo effects at the mode.

\subsection{Determining the Typical Lifespan and Compression of Deaths above the Mode}

Although conceptually straightforward, finding the modal age at death may, in practice, be complicated by irregularities of the life table distribution of deaths $d(x)$ and need for computing the mode in fractions of year while using discreet life table. Kannisto (2001) discusses the problem and proposes a formula based on quadratic approximation to $d(x)$ to be applied after a not-'excessive' smoothing of the distribution. He warns against excessive smoothing of the curve, which may reduce the height of the mode and affect findings concerning compression of deaths.

The key relationship, in any of its equivalent forms, provides an alternative approach. One may use, for example, the following discrete approximation to Eq. (4):

$$
\frac{\ln M_{x+1}-\ln M_{x-1}}{2}-M_{x}=0,
$$

where the expression in the left-hand side may be smoothed and numerically resolved by any convenient procedure, which will not affect considerably the distribution of deaths, as the expression is a monotonic function of age (this is what we use in the following illustrations).

Solution to Eq. (1) is also informative about compression of deaths above the mode. Assuming, in line with Lexis and Kannisto, that 'natural' deaths due to senescence are, roughly, normally distributed and there are no premature deaths above the mode, one can expect tight link between the mortality rate at the mode and the standard deviation of age at death above the mode. In the normal distribution, the root mean square deviation from the mode is $\frac{2}{\sqrt{2 \pi}} \approx 0.80$ times $\frac{1}{\mu(M)}$. This ratio is close to the empirical ratio 0.74 (with sample standard deviation 0.02) that we obtain from distributions of deaths above the modal age in the Human Mortality Database (2011). The standard deviation from the mode of age at death above the mode may then be approximated as

$$
S D(M+) \approx \frac{0.74}{\mu(M)}(\text { years }) \text {. (9) }
$$

Numerical illustrations are presented in Table 1 summarizing estimates of the mode and of the standard deviation of age at death above the mode in period life tables for currently low-mortality countries. The standard deviations are presented in two forms: estimated 
directly from the period life table distributions and indirectly, using approximation (9). The table also features mean absolute and mean absolute percentage errors of Eq. (9) (about 0.3 years and 5\%, respectively). Good accordance between the direct estimates and the approximation indicates efficiency of the latter. The table illustrates well-reported phenomenon of mortality compression above the mode in period life tables accompanying increase in the mode itself (e.g., Kannisto 2000, 2001, Canudas-Romo 2008, Thatcher et al. 2010).

Hence, the key relation (1) and its equivalents tell the two most important stories about 'natural' adult deaths. First, the age $M$ fulfilling the relation indicates the typical natural life duration. Second, the mortality level $\mu(M)$ balancing the equations indicates variability of natural age at death.

Table 1. Modal age at death and standard deviation of age at death above the mode in period life tables averaged over currently low-mortality countries in selected periods, years. Numbers in the parentheses indicate inter-country standard deviation of an indicator.

\begin{tabular}{|c|c|c|c|c|}
\hline Period & $\begin{array}{c}\text { Before } \\
1900\end{array}$ & 1900-1945 & 1946-1979 & $\begin{array}{l}1980 \text { and } \\
\text { later }\end{array}$ \\
\hline \multicolumn{5}{|l|}{ Females: } \\
\hline Modal age at death & $74.4(2.7)$ & $77.4(2.0)$ & $81.6(2.0)$ & $86.5(2.0)$ \\
\hline $\mathbf{S D}(\mathbf{M}+)$, direct estimate & $8.5(0.8)$ & $7.9(0.7)$ & $7.1(0.6)$ & $6.1(0.6)$ \\
\hline SD(M+), approximated from Eq. (9) & $8.3(1.0)$ & $7.8(0.9)$ & $7.1(0.7)$ & $6.4(0.6)$ \\
\hline Mean absolute error of Eq. (9) & 0.4 & 0.3 & 0.2 & 0.3 \\
\hline $\begin{array}{r}\text { Mean absolute percentage error of } \\
\text { Eq. (9) }\end{array}$ & $4.5 \%$ & $3.9 \%$ & $2.2 \%$ & $5.7 \%$ \\
\hline \multicolumn{5}{|l|}{ Males: } \\
\hline Modal age at death & $73.2(2.7)$ & $75.9(2.6)$ & $77.2(1.9)$ & $81.5(2.5)$ \\
\hline $\mathbf{S D}(\mathbf{M}+)$, direct estimate & $8.7(0.9)$ & $7.9(0.8)$ & $8.2(0.7)$ & $7.4(0.7)$ \\
\hline SD(M+), approximated from Eq. (9) & $8.5(1.2)$ & $7.9(1.1)$ & $8.2(0.9)$ & $7.5(0.8)$ \\
\hline Mean absolute error of Eq. (9) & 0.5 & 0.3 & 0.2 & 0.2 \\
\hline Mean absolute percentage error of & $5.3 \%$ & $3.6 \%$ & $2.5 \%$ & $2.8 \%$ \\
\hline
\end{tabular}

Data sources: Human Mortality Database (2011) period life tables for currently low-mortality countries/territories (Australia, Austria, Belgium, Canada, Chile, Denmark, Finland, France, Ireland, Israel, Italy, Japan, Netherlands, New Zealand, Norway, Portugal, Spain, Sweden, Switzerland, Taiwan, USA, UK, West Germany)

\subsection{Application in the Sensitivity Analysis}

The relationships presented above are valuable in studying effects of gradual mortality change on the mode and compression of deaths above it.

Consider, first, a simple, though unrealistic, scenario of proportionate change of the hazard function (in the text below, the cup sign denotes indicators of the new mortality regime; indicators without the cup are those of the regime before the mortality change):

$\hat{\mu}(x)=e^{-b} \mu(x),(10)$

where $b$ is a (small) parameter of mortality change; death rates decline at positive $b$ and increase at negative $b$. Substituting this into (1) at the new mode yields, at $\alpha=\alpha^{*}$ (note usage of approximate constancy of the left-hand side in (1) at this value of $\alpha$ ): 


$$
\begin{gathered}
\hat{\mu}(\hat{M})=\sqrt[2-\alpha]{\frac{1}{1-\alpha} \frac{d}{d x} \hat{\mu}^{1-\alpha}(\hat{M})=} e^{-\frac{1-\alpha}{2-\alpha} b} \sqrt[2-\alpha]{\frac{1}{1-\alpha} \frac{d}{d x} \mu^{1-\alpha}(\hat{M})} \approx e^{-\frac{1-\alpha}{2-\alpha} b} \sqrt[2-\alpha]{\frac{1}{1-\alpha} \frac{d}{d x} \mu^{1-\alpha}(M)}= \\
=e^{-\frac{1-\alpha}{2-\alpha} b} \mu(M) .(11)
\end{gathered}
$$

Applying (10) to the left-hand side in (11), expanding the first-order approximation and using (3):

$$
\hat{\mu}(\hat{M})=e^{-b} \mu(\hat{M}) \approx e^{-b}\left(\mu(M)+\frac{d \mu}{d x}(M) \cdot(\hat{M}-M)\right)=e^{-b}\left(\mu(M)+\mu^{2}(M) \cdot(\hat{M}-M)\right) .
$$

Equating this to the right-hand side in (11), resolving for $\hat{M}$, substituting $\alpha=\alpha^{*} \approx 0.8$ and using (9), the change in the mode may, finally, be derived:

$$
\hat{M}=M+\frac{e^{\frac{1}{2-\alpha} b}-1}{\mu(M)} \approx M+\frac{1}{2-\alpha} \frac{b}{\mu(M)} \approx M+1.1 \cdot S D(M+) \cdot b \text {. }
$$

From (11) and (9), we may also infer the effect on the mortality compression above the mode:

$$
\hat{S} D(\hat{M}+) \approx e^{\frac{1-\alpha}{2-\alpha}} S D(M+) \approx\left(1+\frac{1-\alpha}{2-\alpha} b\right) S D(M+) \approx(1+0.17 b) \cdot S D(M+) .
$$

Hence, proportionate mortality decline increases the mode and results in mortality decompression above the mode; $1 \%$ proportionate mortality decline would be accompanied by $0.17 \%$ decompression and shift the mode by about $1.1 \%$ of $S D(M+)$.

As readily seen from Table 1, however, proportionate decline of mortality does not describe well mortality change in period life tables, where mortality decline was accompanied by compression of deaths above the mode. Unlike in the simplistic model above, data show mortality decline varying with age, which may be described by a more realistic model where the small parameter $b$ of mortality change is age-specific:

$$
\hat{\mu}(x)=e^{-b(x)} \mu(x) \text {. (15) }
$$

Typically, the speed of mortality change is higher at younger adult ages than at older ages, i.e. $\frac{d b(x)}{d x}$ and $b(x)$ are of opposite sign around the mode.

Using scenario (15), one may derive from (1), (9), at $\alpha=\alpha^{*} \approx 0.8$, the following firstorder approximations (detailed derivations may be found in the Appendix):

$$
\begin{aligned}
& \hat{M} \approx M+\frac{1}{2-\alpha} \frac{b(M)}{\mu(M)}-\frac{1}{2-\alpha} \frac{\frac{d b}{d x}(M)}{\mu^{2}(M)} \approx \\
& \approx M+S D(M+)\left(1.1 b(M)-1.5 S D(M+) \cdot \frac{d b}{d x}(M)\right) . \\
& \begin{aligned}
\hat{S} D(\hat{M}+) \approx S D(M+) & \left(1+\frac{1-\alpha}{2-\alpha} b(M)+\frac{1}{2-\alpha} \frac{\frac{d b}{d x}(M)}{\mu(M)}\right) \approx \\
& \approx S D(M+)\left(1+0.17 b(M)+1.1 S D(M+) \cdot \frac{d b}{d x}(M)\right) .
\end{aligned}
\end{aligned}
$$

Given opposite signs of $\frac{d b(x)}{d x}$ and $b(x)$, (16) will produce larger shifts of the modal age as compared to the proportionate mortality change case (13). The standard deviation (17) may also look very different from (14) showing decompression or compression of deaths depending on the balance between the summands of opposite sign in (17). Based on HMD data for low mortality countries since 1980 , we estimate $b(M) \approx 0.016$ with age derivative $\frac{d}{d x} b(M) \approx-0.00087$ (averaged over the entire period and both sexes). Substituting these estimates into (16), (17) and assuming, roughly, $S D(M+) \approx 7$ years, yields the mode's shift of 0.2 years per year and mortality compression by about $0.4 \%$ per year. This is fairly 
consistent with data showing 0.2 years per year increase of the mode and $0.7 \%$ annual compression of mortality above the mode.

\subsection{Modal Age at Death and Mortality Compression in Birth Cohorts}

A particularly important application of the relations presented is to study the mode and mortality compression above the mode in birth cohorts. Involving mortality rates over only a few years of life of the cohort around the mode, the relations do away with the main obstacle (incomplete observation of cohorts' life history) which has so far prevented the study of recent developments of mortality compression in birth cohorts.

Simultaneously, the above equations ascertain that cohort mortality schedules will demonstrate mortality compression effects different from the results reported for period life tables when the modal age changes over time. While the force of mortality $\mu(x)$ is similar from period and cohort perspectives, the left-hand side in (1) is different in period mortality schedules as compared to cohorts reaching the given age $x$ in the given period. This is because of the tempo effect: with increasing age at a certain mortality level, period life tables report shorter exposure durations to small ranges of the mortality rate around the given level (i.e. a steeper increase of the mortality rate and higher derivatives in the left-hand side of (1)); vice versa for decreasing age at the given mortality level (Ediev 2008, 2011). It therefore follows for the mortality decreasing with time:

(i) the period modal age $M_{p}(t)$ at a given period $t$ will be systematically higher than the cohort modal age $M_{c}\left(t-M_{p}(t)\right)$ for the cohort aged $M_{p}(t)$ in the given period;

(ii) with the modal age in the cohort $t-M_{p}(t)$ being younger than $M_{p}(t)$, Eq. (1) will balance at a lower mortality rate and deaths will be less compressed as compared to period $t$;

(iii)deaths will also be less compressed in the cohort, which will have the same modal age at death $M_{p}(t)$ as in the period $t$, because Eq. (1), being fulfilled for the cohort at age $M_{p}(t)$ at some moment $t^{\prime}>t$ in the future, will balance at a lower (due to decreasing mortality) force of mortality.

Numerical results in Table 2, produced in the same format as Table 1 above, illustrate these points. Before 1900, when mortality decline was rather small and the tempo-effect was minor, both cohorts and period life tables showed similar modes and compression indicators. In the period that followed, mortality decline gained momentum and the tempo effect became sizable. Period cross-sections indicated rapid increase of the modal age at death accompanied by the mortality compression above it, while birth cohorts showed systematically lower mode and less compression. For males, despite overall mortality decline since 1900, there was rather a de-compression of deaths above the mode. 
Table 2. Modal age at death and standard deviation of age at death above the mode in cohort life tables averaged over currently low-mortality countries in selected periods, years. Numbers in the parentheses indicate inter-country standard deviation of an indicator.

\begin{tabular}{|c|c|c|c|c|}
\hline Period when the mode is reached & $\begin{array}{c}\text { Before } \\
1900\end{array}$ & $1900-1945$ & 1946-1979 & $\begin{array}{l}1980 \text { and } \\
\text { later }\end{array}$ \\
\hline \multicolumn{5}{|l|}{ Females: } \\
\hline Modal age at death & $74.3(2.7)$ & $77.1(2.4)$ & $80.7(1.8)$ & $84.3(1.9)$ \\
\hline $\mathbf{S D}(\mathbf{M}+)$, direct estimate & $8.6(0.8)$ & $8.1(0.9)$ & $7.8(0.7)$ & n.a. \\
\hline $\mathbf{S D}(\mathbf{M}+)$, approximated from Eq. (9) & $8.4(1.1)$ & $8.0(1.2)$ & $7.9(0.9)$ & $7.3(0.8)$ \\
\hline Mean absolute error of Eq. (9) & 0.4 & 0.4 & 0.3 & \\
\hline $\begin{array}{r}\text { Mean absolute percentage error of } \\
\text { Eq. (9) }\end{array}$ & $4.9 \%$ & $4.8 \%$ & $3.5 \%$ & \\
\hline \multicolumn{5}{|l|}{ Males: } \\
\hline Modal age at death & $73.1(2.8)$ & $75.6(2.7)$ & $76.8(2.1)$ & $77.7(1.7)$ \\
\hline $\mathbf{S D}(\mathbf{M}+)$, direct estimate & $8.7(0.9)$ & $8.2(0.9)$ & $8.5(0.9)$ & n.a. \\
\hline SD(M+), approximated from Eq. (9) & $8.7(1.4)$ & $8.1(1.3)$ & $8.5(1.0)$ & $9.1(0.9)$ \\
\hline Mean absolute error of Eq. (9) & 0.4 & 0.4 & 0.2 & \\
\hline $\begin{array}{r}\text { Mean absolute percentage error of } \\
\text { Eq. (9) }\end{array}$ & $4.8 \%$ & $5.1 \%$ & $2.8 \%$ & \\
\hline
\end{tabular}

Data source: Human Mortality Database (2011) cohort mortality rates for currently lowmortality countries/territories (Australia, Austria, Belgium, Canada, Denmark, Finland, France, Ireland, Italy, Japan, Netherlands, New Zealand, Norway, Portugal, Spain, Sweden, Switzerland, Taiwan, USA, UK, West Germany)

One can assess cohort-period differentials in mortality compression analytically. Differentiating the mortality rate at the period modal age by time and using (3) yields:

$$
\begin{gathered}
\frac{d}{d t} \mu\left(M_{p}(t), t\right)=\frac{\partial}{\partial x} \mu\left(M_{p}(t), t\right) \frac{d}{d t} M_{p}(t)+\frac{\partial}{\partial t} \mu\left(M_{p}(t), t\right)=\frac{\partial}{\partial x} \mu\left(M_{p}(t), t\right)\left(\frac{d}{d t} M_{p}(t)-r\left(M_{p}(t), t\right)\right) \\
=\mu^{2}\left(M_{p}(t), t\right)\left(\frac{d}{d t} M_{p}(t)-r\left(M_{p}(t), t\right)\right),(18)
\end{gathered}
$$

where $r\left(M_{p}(t), t\right)=-\frac{\frac{\partial}{\partial t} \mu\left(M_{p}(t), t\right)}{\frac{\partial}{\partial x} \mu\left(M_{p}(t), t\right)}$ is the tangent slope at time $t$ of the contour line corresponding to the mortality level $\mu=\mu\left(M_{p}(t), t\right)$ (the change rate of the age at which mortality level is fixed at $\left.\mu=\mu\left(M_{p}(t), t\right)\right)$; this particular rate determines the tempo effect in that the period life tables report exposures to small ranges of the mortality rate compressed by $\frac{1}{1-r}$ times as compared to the cohort exposures around the mode (Ediev 2008, 2011). Resolving (18) for $r\left(M_{p}(t), t\right)$,

$$
r\left(M_{p}(t), t\right)=\frac{d}{d t} M_{p}(t)+\frac{d}{d t} \mu^{-1}\left(M_{p}(t), t\right)
$$

Hence, the cohort-wise derivative in (4) will be $(1-r(\cdot))$ times the period-wise derivative, and relation (1) will be balanced for cohorts at mortality rate by about

$$
\frac{\mu\left(M_{c}\right)}{\mu\left(M_{p}\right)}=\sqrt[2-\alpha^{*}]{1-r} \approx 1-\frac{r}{2-\alpha^{*}} \approx 1-0.83 r
$$

times the balancing rate for period life tables (mind the choice $\alpha^{*} \approx 0.8$, which makes the left-hand side in (1) approximately constant around the mode). Consequently, the cohort distribution of age at natural death shall be $\frac{1}{1-0.83 r}$ times less compressed as compared to the period distribution. Since the magnitude of the difference in compression will vary depending on the speed of mortality decline, periods and cohorts may show different trends of the mortality compression. 
As an empirical illustration, consider the mortality data for France in the years 1977, 2007 and cohorts born in 1886 and 1916 (HMD 2010). For females, the period modal age at adult death has increased in the period of 30 years from about 84.7 to 90.4 years and the mortality rate at the mode (changed from about 110 to 125 per 1,000) indicates a mortality compression (also note, from Eq. (19), $r=\frac{90.4-84.7+0.125^{-1}-0.110^{-1}}{30}=0.15$ years per year). The cohort modal age changed from about 82.3 to 89.0 and mortality rates at the cohort modal age at death (changed from about 99 to 114 per 1,000) were by about $1-0.83 r$ times lower than the period rates but also indicate a compression. For males, the period modal age increased from about 78.2 to 86.2 years and the mortality rate at the period mode (increased from 87 to 112 per 1,000$)$ indicates mortality compression $\left(r=\frac{86.2-78.2+0.112^{-1}-0.087^{-1}}{30}=0.18\right.$ years per year). Males' cohort modal age at death has also increased (77.0 to 82.2 years) but the mortality rate at the mode (90 per 1,000 in both cohorts) was about $1-0.83 r$ times lower than the corresponding period rate only in the more recent life table and indicated no considerable compression. (The tempo effect did not result in the difference between cohort and period rates for the earlier life tables, because the decline of males' mortality rates around the mode started later.) 


\section{References}

Canudas-Romo, V. (2008). The modal age at death and the shifting mortality hypothesis. Demographic Research, 19(30):1179-1204. (http://www.demographic-research.org/Volumes/Vol19/30/19-30.pdf.)

Carey, J.R. and Liedo, P. (1995). Sex-specific life table aging rates in large medfly cohorts. Experimental Gerontologist 30:315-325.

Cheung, S. L. K. and Robine, J.-M. (2007). Increase in common longevity and the compression of mortality: The case of Japan. Population Studies, 61(1):85-97.

Ediev, D.M. (2008). On the Theory of Distortions of Period Estimates of the Quantum Caused by the Tempo Changes. European Demographic Research Paper 3/2008. Vienna Institute of Demography, Austrian Academy of Sciences. (http://www.oeaw.ac.at/vid/download/edrp_3 08.pdf.)

Ediev, D.M. (2011). Life Expectancy in Developed Countries Is Higher Than Conventionally Estimated. Implications from Improved Measurement of Human Longevity. Journal of Population Ageing. Published online ahead of print. (http://www.springerlink.com/content/lu61j0v1k1951253/)

Fries, J. (1980). Aging, natural death, and the compression of morbidity. New England Journal of Medicine, 303(3):130-135.

HMD. (2011). Human Mortality Database sponsored by the University of California, Berkeley (USA) and the Max Planck Institute for Demographic Research (Germany). «www.mortality.org» or «www.humanmortality.de». Accessed on 19-04-2011.

Kannisto, V. (2000). Measuring the compression of mortality. Demographic Research, 3(6). (http://www.demographic-research.org/Volumes/Vol3/6/3-6.pdf.)

Kannisto, V. (2001). Mode and dispersion of the length of life. Population: An English Selection, 13:159-171.

Lexis, W. (1878). Sur la durée normale de la vie humaine et sur la théorie de la stabilité des rapports statistiques. [On the normal human lifespan and on the theory of the stability of the statistical ratios]. Annales de Démographie Internationale, 2:447-460.

Pollard, J. H. (1991). Fun with Gompertz. Genus, 47(1-2):1-20.

Schoen, R. (2006). Dynamic Population Models. Springer, Dordrecht.

Thatcher, A.R., Cheung, S.L.K, Horiuchi, Sh., Robine, J.-M. (2010). The compression of deaths above the mode. Demographic Research, 22(17):505-538. (http://www.demographic-research.org/Volumes/Vol22/17/22-17.pdf)

Tuljapurkar, Sh. and Edwards, R. D. (2011). Variance in death and its implications for modeling and forecasting mortality. Demographic Research, 24(21): 497-526. (http://www.demographic-research.org/volumes/vol24/21/24-21.pdf.)

Wilmoth, J. and Horiuchi, S. (1999). Rectangularization revisited: Variability of age at death within human populations. Demography, 36(4):475-495. 


\section{Appendix: Derivation of First-Order Approximations (16), (17)}

Substituting Eq. (15) into (1) at the new mode yields, at $\alpha=\alpha^{*}$ :

$$
\begin{aligned}
\hat{\mu}(\hat{M})=\sqrt[2-\alpha]{\frac{1}{1-\alpha} \frac{d \hat{\mu}^{1-\alpha}}{d x}}(\hat{M}) & =\sqrt[2-\alpha]{\frac{1}{1-\alpha} \frac{d}{d x}\left[e^{-b(x)(1-\alpha)} \mu^{1-\alpha}(x)\right]_{x=\hat{M}}}= \\
& =\sqrt[2-\alpha]{\frac{1}{1-\alpha}\left[-(1-\alpha) e^{-b(x)(1-\alpha)} \mu^{1-\alpha}(x) \frac{d}{d x} b(x)+e^{-b(x)(1-\alpha)} \frac{d}{d x} \mu^{1-\alpha}(x)\right]_{x=\hat{M}}}=
\end{aligned}
$$

/rearranging the expression, expanding the Taylor series and dropping second-order summands in- and out-side the power-function/

$$
=2-\alpha \frac{1}{1-\alpha} e^{-b(\hat{M})(1-\alpha)} \frac{d \mu^{1-\alpha}}{d x}(\hat{M})\left[1-\frac{(1-\alpha) \mu^{1-\alpha}(\hat{M}) \frac{d b}{d x}(\hat{M})}{\frac{d \mu^{1-\alpha}}{d x}(\hat{M})}\right] \approx \sqrt[2-\alpha]{\frac{1}{1-\alpha} e^{-b(M)(1-\alpha)} \frac{d \mu^{1-\alpha}}{d x}(\hat{M})}\left[1-\frac{(1-\alpha) \mu^{1-\alpha}(M) \frac{d b}{d x}(M)}{(2-\alpha) \frac{d 1^{1^{-\alpha}} d x}{d x}(M)}\right]=
$$

/rearranging and using approximate constancy of the left-hand side in (1) at $\alpha=\alpha^{*} /$

$$
=e^{-\frac{1-\alpha}{2-\alpha} b(M)} \sqrt[2-\alpha]{\frac{1}{1-\alpha} \frac{d \mu^{1-\alpha}}{d x}(\hat{M})}\left[1-\frac{(1-\alpha) \mu^{1-\alpha}(M) \frac{d b}{d x}(M)}{(2-\alpha) \frac{d \mu^{1-\alpha}}{d x}(M)}\right] \approx e^{-\frac{1-\alpha}{2-\alpha} b(M)} \sqrt[2-\alpha]{\frac{1}{1-\alpha} \frac{d \mu^{1-\alpha}}{d x}(M)}\left[1-\frac{(1-\alpha) \mu^{1-\alpha}(M) \frac{d b}{d x}(M)}{(2-\alpha) \frac{d \mu^{1}}{d x}(M)}\right]=
$$

/applying (1)/

$$
=\mu(M) e^{-\frac{1-\alpha}{2-\alpha} b(M)}\left[1-\frac{(1-\alpha) \mu^{1-\alpha}(M) \frac{d b}{d x}(M)}{(2-\alpha)(1-\alpha) \mu^{2-\alpha}(M)}\right]=\mu(M) e^{-\frac{1-\alpha}{2-\alpha} b(M)}\left[1-\frac{\frac{d b}{d x}(M)}{(2-\alpha) \mu(M)}\right] \approx
$$

/expanding the Taylor series and parentheses and dropping second-order summands/

$$
\approx \mu(M)\left[1-\frac{1-\alpha}{2-\alpha} b(M)\right]\left[1-\frac{\frac{d b}{d x}(M)}{(2-\alpha) \mu(M)}\right] \approx \mu(M)\left[1-\frac{1-\alpha}{2-\alpha} b(M)-\frac{\frac{d b}{d x}(M)}{(2-\alpha) \mu(M)}\right] .
$$

Applying (15) and expanding the first-order approximation to the left-hand side in (A1) and using (3):

$$
\begin{gathered}
\hat{\mu}(\hat{M})=e^{-b(\hat{M})} \mu(\hat{M}) \approx e^{-b(M)}\left(\mu(M)+\frac{d \mu}{d x}(M) \cdot(\hat{M}-M)\right)= \\
=e^{-b(M)}\left(\mu(M)+\mu^{2}(M) \cdot(\hat{M}-M)\right) \approx \\
\approx[1-b(M)]\left[\mu(M)+\mu^{2}(M) \cdot(\hat{M}-M)\right] \approx \mu(M)[1-b(M)+\mu(M) \cdot(\hat{M}-M)] .
\end{gathered}
$$

Equating this expression to the right-hand side in (A1), resolving for $\hat{M}$, substituting $\alpha=\alpha^{*} \approx 0.8$ and using (9) yields Eq. (16).

Eq. (17) follows directly from (A1) and (9). 


\section{VIENNA INSTITUTE OF DEMOGRAPHY}

\section{Working Papers}

Zeman, Kryštof, Tomáš Sobotka, Richard Gisser, Maria Winkler-Dworak, and Wolfgang Lutz, Geburtenbarometer Vienna: Analysing Fertility Convergence between Vienna and Austria (English version and German version), VID Working Paper 06/2011.

Feichtinger, Gustav, Michael Kuhn, Alexia Prskawetz, and Stefan Wrzaczek, The Reproductive Value as Part of the Shadow Price of Population, VID Working Paper 05/2011.

Barakat, Bilal, Time is Money: Could Deferred Graduate Retirement Finance Higher Education? VID Working Paper 04/2011.

Riosmena, Fernando, Maria Winkler-Dworak, Alexia Prskawetz, and Gustav Feichtinger, The Impact of Policies Influencing the Demography of Age Structured Populations: Lessons from Academies of Science, VID Working Paper 03/2011.

Buber, Isabella and Katrin Fliegenschnee, Are you Ready for a Child? A Methodological Triangulation on Fertility Intentions in Austria, VID Working Paper $02 / 2011$.

Fent, Thomas, Belinda Aparicio Diaz, and Alexia Prskawetz, Family Policies in the Context of Low Fertility and Social Structure, VID Working Paper 01/2011.

Buber, Isabella, Caroline Berghammer, and Alexia Prskawetz, Doing Science, Forgoing Childbearing? Evidence from a Sample of Female Scientists in Austria, VID Working Paper 09/2010.

Strulik, Holger, Klaus Prettner and Alexia Prskawetz, R\&D-Based Growth in the Post-Modern Era, VID Working Paper 08/2010.

Prettner, Klaus and Alexia Prskawetz, Demographic Change in Models of Endogenous Economic Growth. A Survey, VID Working Paper 07/2010.

Flandorfer, Priska, Christian Wegner, and Isabella Buber, Gender Roles and Smoking Behaviour, VID Working Paper 06/2010.

Barakat, Bilal, Johannes Holler, Klaus Prettner, and Julia Schuster, The Impact of the Economic Crisis on Labour and Education in Europe, VID Working Paper 05/2010

Grafeneder-Weissteiner, Theresa and Klaus Prettner, Agglomeration Processes in Ageing Societies, VID Working Paper 04/2010.

The Vienna Institute of Demography Working Paper Series receives only limited review. Views or opinions expressed herein are entirely those of the authors. 